\title{
The Short Term Load Forecasting of RBF Neural Network Power System Based on Fuzzy Control
}

\author{
Jiangnan Ni 1, Guo Jin 2 \\ ${ }^{1}$ College of mechanical and electrical automation, Henan polytechnic institute, Henan Nanyang \\ 473000, China
}

Keywords: Power system, Load forecasting, RBF neural network, Fuzzy control.

\begin{abstract}
This paper presents a kind of power system short-term load prediction algorithm based on fuzzy control and RBF neural network, to solve the problems of th traditional RBF neural network in electric power system short-term load forecast errors. Through the example verification, this method can improve the prediction accuracy compared with the traditional RBF load forecasting method, which has a good application prospect.
\end{abstract}

\section{Introduction}

In recent years, with the continuous introduction of competition mechanism, the power system in our country is gradually transition from the monopoly operation stage to the power generation competition stage, and the work of load forecasting has changed gradually [1-3]. The importance of load forecasting has been widespread attention, the way of artificial prediction has replaced by artificial way of previous prediction for the method of load forecasting, so load forecasting software has become an important part of energy management system (EMS). In addition, due to the popularity of the computer, a large number of load forecasting theory algorithm and prediction model can be used in the power market environment [4-6]. At present, there are many methods in power system short-term load forecasting system, a neural network and fuzzy theory prediction methods are highly valued by researchers at home and abroad, and it is considered to be an effective method [7,8]. Under this background, this paper has carried on the thorough discussion in view of all kinds of problems of the electric power system short-term load forecasting system, this paper establishes short term load forecasting model based on the combination of fuzzy control theory and RBF neural network, to deal with the historical load data and the load influence factors. Finally, the examples verify the accuracy of the prediction model.

\section{RBF Neural Network Architecture}

The neural network structure of radial basis function (RBF) includes 3 layers, the left, middle and right order are respectively the input node, hidden node and output point, and its network structure is shown in Figure 1. Each layer has a completely different role: the input layer is composed of the signal source node, its role is to take the input signal and its transfer to the hidden layer; hidden layer is formed by a Gaussian kernel function as radiation shape function, which is one of the most important layer in RBF network; output layer is a linear combination of the nonlinear basis functions of the hidden layer nodes, so we can get the final result.

The mathematical description of the RBF network can be expressed as: given $N$ input samples is $x_{i}=(\mathrm{i}=1,2, \cdots, N)$ in $n$-dimensional space, and then the output of the $k$-th node in the network hidden layer can be expressed as:

$$
r_{k}=\mathbb{R}\left(\left\|x_{i}-T_{k}\right\|\right) \text {. }
$$

Among them, $x_{i}$ represents the n-dimensional input vector; $T_{k}$ represents the center of the $k$-th hidden node, in which $k=1,2, \cdots, 1 ;\|\cdot\|$ is usually the European norm; $R(\cdot)$ represents the RBF function, it has the characteristic of local feeling, and it reflects the ability of nonlinear mapping of RBF network. In the network output layer, the output of the $j$-th node is a linear mapping of the hidden layer node to the output layer, namely: 


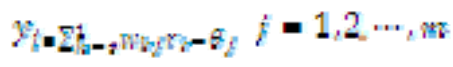

Among them, $w_{k j}$ represents the value of the hidden layer to the output layer; $\theta_{j}$ is the threshold of the $j$-th output node; $m$ represents the number of nodes in the output layer.

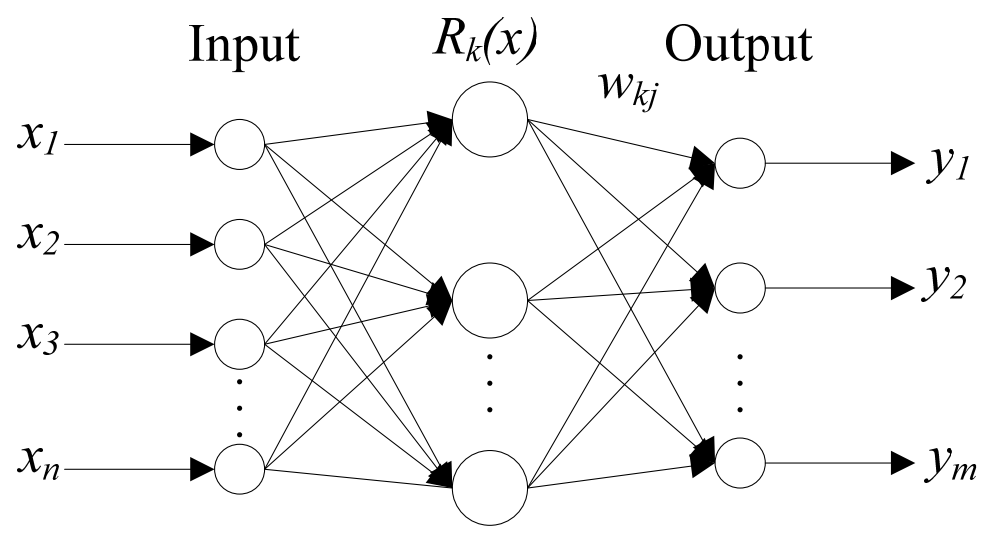

Fig.1 RBF network structure diagram

\section{The Short Term Load Algorithm of RBF Neural Network Power System Based on Fuzzy Control}

With the change of power system load, the internal relationship between RBF neural network should also be changed. If the use of the original neural network parameters carry out load forecasting, it tends to produce large errors, but fuzzy adjustment can change this kind of phenomenon, it can improve the load forecasting accuracy. In the short-term load forecasting of power system, this paper can carry out an online self tuning fuzzy control based on RBF neural network prediction [9-11]. Assume that the output of the RBF neural network is $U$, fuzzy adjustmen output is $\Delta U$, the final output of the network prediction is $Y=U+\Delta U$, and its prediction process is shown in Figure 2.

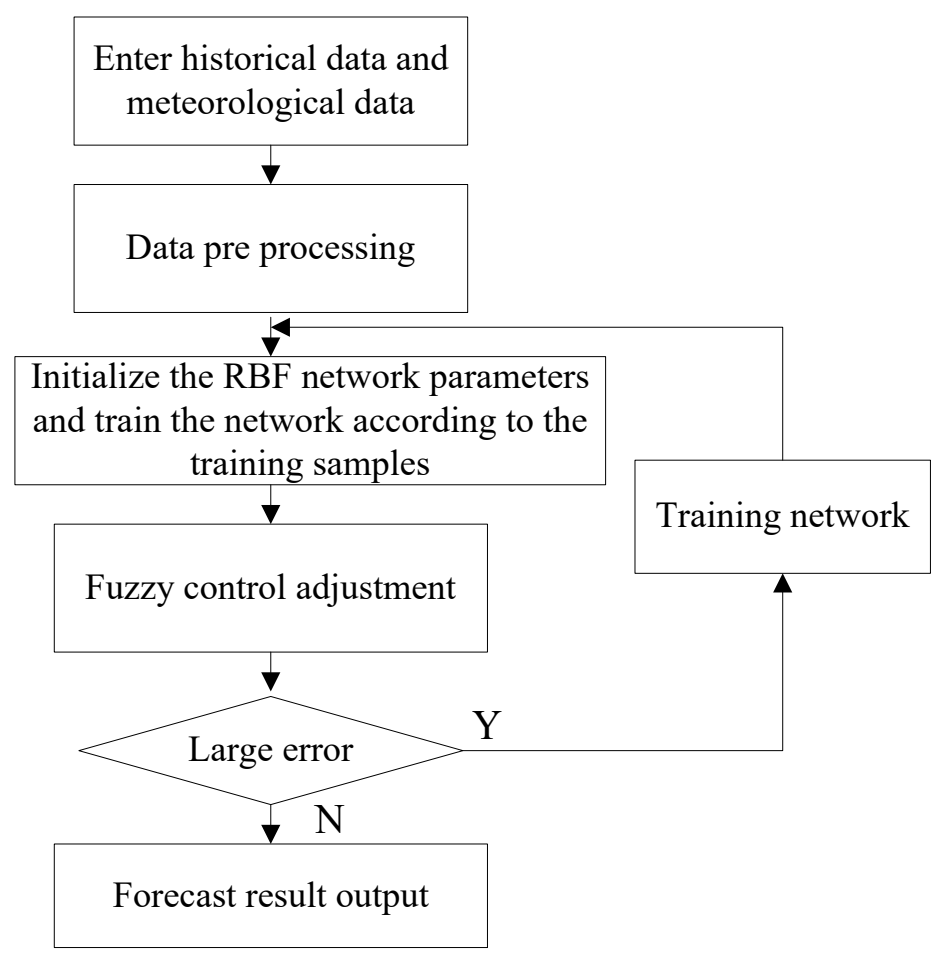

Fig. 2 Fuzzy control RBF network prediction system workflow 


\section{The Example Analysis of Short Term Load Forecasting in Power System}

In order to verify the validity and accuracy of the method, this paper selects the power load of a certain area in Henan province. This paper establishes 24 RBF neural network prediction model, the input layer node of each neural network is 12 , the hidden layer node is 16 and the output layer node is 1. The proposed algorithm is compared with the traditional RBF neural network algorithm, its load forecasting curve is shown in Figure 3, and the relative error curve is shown in Figure 4. The prediction data of RBF and fuzzy control RBF model are compared, and the results are shown in Table 1.

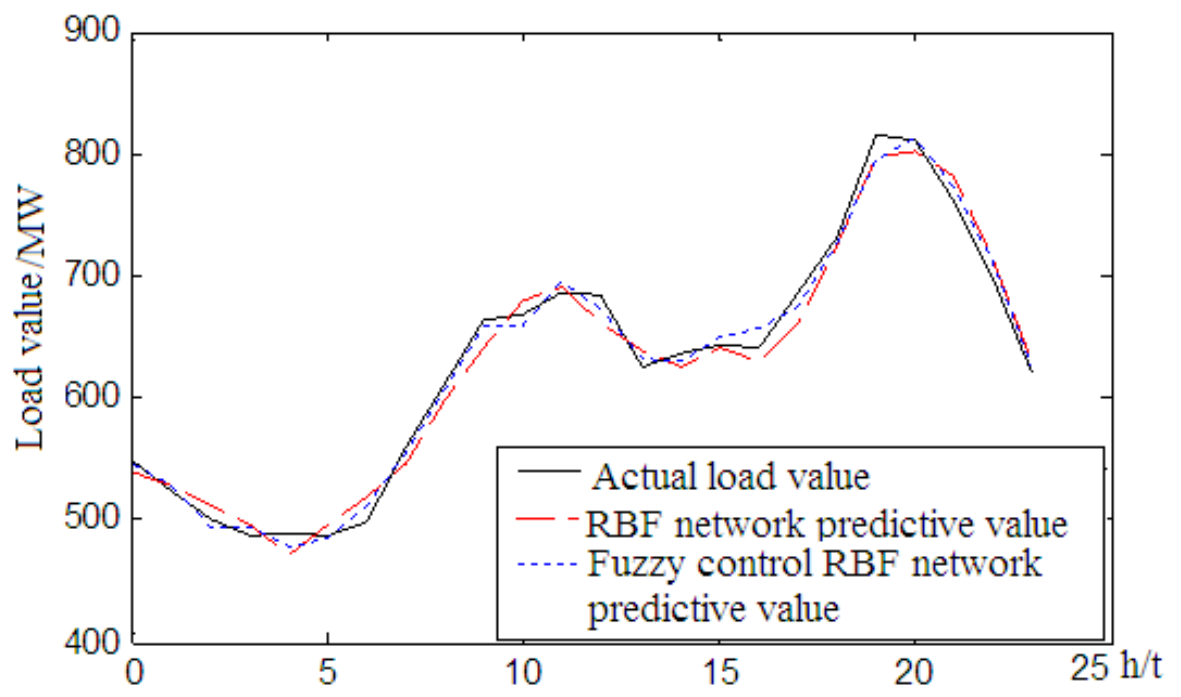

Fig. 3 The load forecasting curve of RBF and fuzzy control RBF model

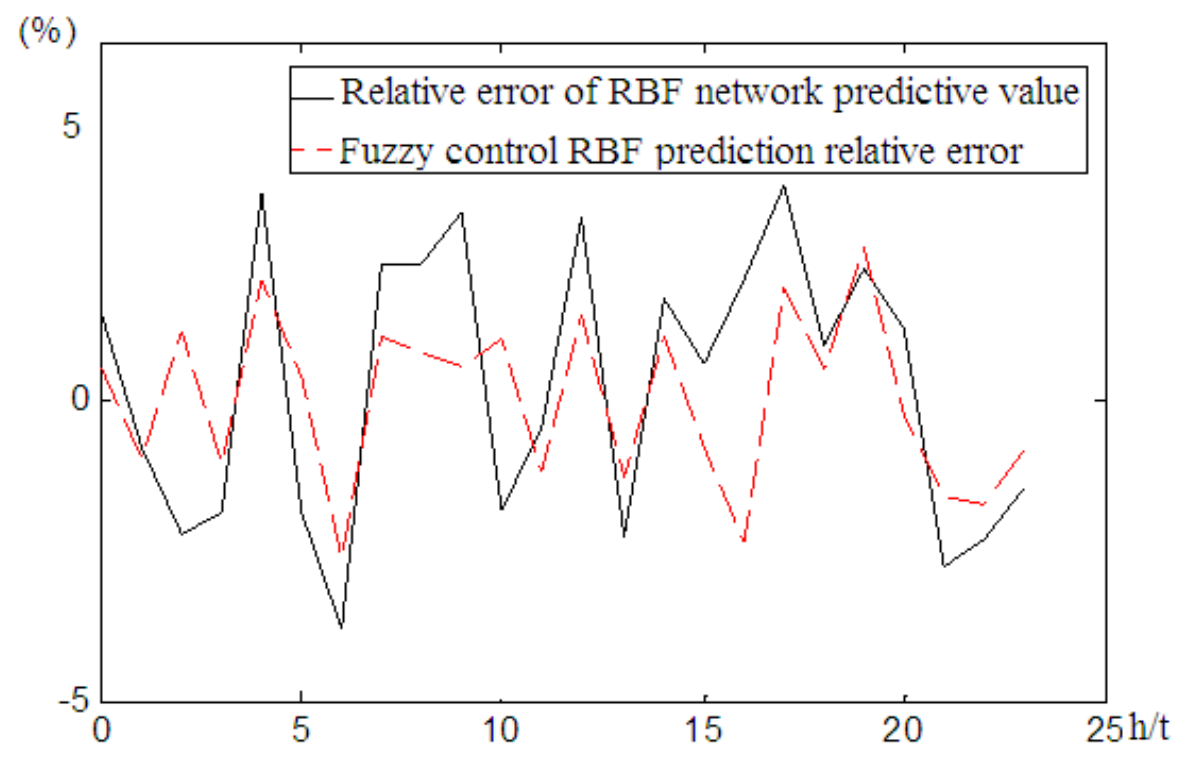

Fig. 4 The load forecast relative error curve of RBF and fuzzy control RBF model

Table 1. The prediction data comparison of RBF and fuzzy control RBF model

\begin{tabular}{|c|c|c|c|c|c|}
\hline \multirow{2}{*}{ Time } & \multirow{2}{*}{$\begin{array}{c}\text { Overload } \\
\text { actual } \\
\text { value }\end{array}$} & \multicolumn{2}{|c|}{$\begin{array}{c}\text { RBF neural network predictive } \\
\text { value }\end{array}$} & \multicolumn{2}{c|}{$\begin{array}{c}\text { Predictive value of RBF neural } \\
\text { network with fuzzy control }\end{array}$} \\
\cline { 3 - 6 } & & $\begin{array}{c}\text { Predicted } \\
\text { value }\end{array}$ & $\begin{array}{c}\text { Relative error } \\
(\%)\end{array}$ & predicted value & $\begin{array}{c}\text { Relative error } \\
(\%)\end{array}$ \\
\hline 1 & 548 & 540 & 1.4599 & 545 & 0.5474 \\
\hline 2 & 523 & 527 & -0.7648 & 528 & -0.9560 \\
\hline
\end{tabular}




\begin{tabular}{|c|c|c|c|c|c|}
\hline 3 & 501 & 512 & -2.1956 & 495 & 1.1976 \\
\hline 4 & 488 & 497 & -1.8443 & 493 & -1.0246 \\
\hline 5 & 489 & 472 & 3.4765 & 479 & 2.0450 \\
\hline 6 & 487 & 496 & -1.8480 & 485 & 0.4107 \\
\hline 7 & 499 & 518 & -3.8076 & 512 & -2.6052 \\
\hline 8 & 560 & 547 & 2.3214 & 554 & 1.0714 \\
\hline 9 & 612 & 598 & 2.2876 & 607 & 0.8170 \\
\hline 10 & 663 & 642 & 3.1674 & 659 & 0.6033 \\
\hline 11 & 667 & 679 & -1.7991 & 660 & 1.0495 \\
\hline 12 & 687 & 690 & -0.4367 & 695 & -1.1645 \\
\hline 13 & 683 & 662 & 3.0747 & 673 & 1.4641 \\
\hline 14 & 625 & 639 & -2.2400 & 633 & -1.2800 \\
\hline 15 & 636 & 625 & 1.7296 & 629 & 1.1006 \\
\hline 16 & 644 & 640 & 0.6211 & 649 & -0.7764 \\
\hline 17 & 642 & 629 & 2.0249 & 657 & -2.3364 \\
\hline 18 & 687 & 662 & 3.6390 & 674 & 1.8923 \\
\hline 19 & 731 & 724 & 0.9576 & 727 & 0.5472 \\
\hline 20 & 816 & 798 & 2.2059 & 795 & 2.5735 \\
\hline 21 & 811 & 801 & 1.2330 & 813 & -0.2466 \\
\hline 22 & 761 & 782 & -2.7595 & 773 & -1.5769 \\
\hline 23 & 698 & 714 & -2.2923 & 710 & -1.7192 \\
\hline 24 & 620 & 629 & -1.4516 & 625 & -0.8065 \\
\hline
\end{tabular}

As shown in Table 1, the maximum relative error of RBF neural network prediction is $3.6390 \%$, the minimum relative error is $2.7595 \%$; the maximum relative error of fuzzy control for RBF network prediction is $2.5735 \%$, and the minimum relative error is $2.6052 \%$.

\section{Summary}

In the short-term load forecasting of power system, the traditional RBF network can produce large error, this paper proposes the short-term load forecasting algorithm based on fuzzy control and RBF neural network, and this algorithm is online self-tuning fuzzy control based on the RBF neural network prediction. The results show that compared with the traditional RBF neural network load forecasting method, the method has higher prediction accuracy.

\section{References}

[1] D.X. Niu, S.H. CAO, L. Zhao. Power load forecasting technology and application. Beijing: China electric power press, 1998: 312-316.

[2] D.Q. Zhu, H. Shi. Artificial neural network theory and its application. Beijing: China electric power press, 2002: 212-218.

[3] Franchini M. Use of a genetic algorithm combined with a local search method for the automatic calibration of conceptual rainfall run off models. Hydrological Science Journal, 1996, 41(1):21 - 39.

[4] L. Xu, KRZYZAK A, OJA E. Rival penalized competitive learning for clustering analysis, RBF network and curve detection. IEEE Trans on Neural Networks, 1993, 4(4): 636-649.

[5] H.M. Zhang, Z.N. Wei et al. A short-term load forecasting approach based on PSO support vector machine. Relay, 2014, 34(3): 28-31.

[6] J. Gao. Artificial neural network theory and emulation example.Beijing: Engineer ingIndustry press, 2013: 113-119. 
[7] W. Chen, B. Feng. Simulation study on the parameters optimization of radial basis function neural network based on QPSO algorithm. Computer application, 2015, 26(8),1928-1930.

[8] Q.Y. Cheng, Y.Y. Wang, W.G. Chen. The short-term load forecasting method based on modified principal component analysis. Power system technology, 2015, 29(3): 64-67.

[9] L.X. Yao, L.F. Song, Q.Y. Li. The short-term load forecasting of power system based on fuzzy clustering analysis and BP network. Power system technology, 2015, 29(1): 20-23.

[10] T. Zhang, D.F. Zhao, L. Zhao. The short term load forecasting method based on RBF neural network and expert system. Journal of Xian Jiaotong University, 2013, 35(4): 3-6.

[11] P. Wang, L. Zhang, X.Y. Chen. The short term load forecasting method based on fuzzy clustering and RBF network. Relay, 2015, 34(10): 64-67. 